\title{
Photodetection-induced relative timing jitter in synchronized time-lens source for coherent Raman scattering microscopy
}

\author{
Jiaqi Wang*,† and Ping Qiu ${ }^{\dagger, *}$ \\ ${ }^{*}$ Key Laboratory of Optoelectronic Devices and \\ Systems of Ministry of Education and Guangdong Province \\ College of Optoelectronic Engineering \\ Shenzhen University \\ Shenzhen 518060, China \\ ${ }^{\dagger}$ College of Physics and Energy \\ Shenzhen University \\ Shenzhen 518060, China \\ tpingqiu@szu.edu.cn
}

Received 7 May 2017

Accepted 7 June 2017

Published 30 June 2017

\begin{abstract}
Synchronized time-lens source is a novel method to generate synchronized optical pulses to modelocked lasers, and has found widespread applications in coherent Raman scattering microscopy. Relative timing jitter between the mode-locked laser and the synchronized time-lens source is a key parameter for evaluating the synchronization performance of such synchronized laser systems. However, the origins of the relative timing jitter in such systems are not fully determined, which in turn prevents the experimental efforts to optimize the synchronization performance. Here, we demonstrate, through theoretical modeling and numerical simulation, that the photodetection could be one physical origin of the relative timing jitter. Comparison with relative timing jitter due to the intrinsic timing jitter of the mode-locked laser is also demonstrated, revealing different qualitative and quantitative behaviors. Based on the nature of this photodetection-induced timing jitter, we further propose several strategies to reduce the relative timing jitter. Our theoretical results will provide guidelines for optimizing synchronization performance in experiments.
\end{abstract}

Keywords: Synchronized time-lens source; coherent Raman scattering microscopy.

\$Corresponding author.

This is an Open Access article published by World Scientific Publishing Company. It is distributed under the terms of the Creative Commons Attribution 4.0 (CC-BY) License. Further distribution of this work is permitted, provided the original work is properly cited. 


\section{Introduction}

Two-color synchronized ultrashort laser source is needed for a variety of physical, chemical and biomedical applications, such as pump-probe experiment $^{1}$ and nonlinear optical microscopy. ${ }^{2,3}$ Tightly synchronized mode-locked laser ${ }^{4}$ and synchronized pumped optical parametric oscillators ${ }^{5}$ are commonly used for imaging purposes. Recently, a novel technology, i.e., the synchronized time-lens source has been demonstrated to be a novel solution and especially appropriate for a variety of coherent Raman scattering (CRS) microscopy applications. ${ }^{6-10}$ The time-lens is based on space-time duality and mimics the spatial lens in the time domain. ${ }^{11-13}$ Driven by electrical signals, ultrashort optical pulses from picoseconds to femtoseconds can be directly generated from a continuous-wave (CW) laser, ${ }^{6,14,15}$ with wavelength tunability of $\sim 100 \mathrm{~nm}$. Synchronized time-lens source employs this pulse generation mechanism and directly uses the modelocked laser optical output pulse trains to generate the electrical drive signal, rendering the following synchronization to the mode-locked lasers.

Relative timing jitter, defined as the root mean square value of the temporal deviation of the pulse peak positions between the two synchronized pulsed laser sources, is the key parameter to evaluate the synchronization performance, including the synchronized time-lens source. So far, the relative timing jitter has been experimentally measured to be on the order of tens to hundreds of femtosecond, ${ }^{6,7,10}$ depending on the specific mode-locked lasers used. However, the origins of this relative timing jitter, especially from a theoretical perspective, are not fully determined. This prevents further optimization of the synchronization performance, especially for the femtosecond synchronization applications considering that the femtosecond timelens source has been demonstrated. ${ }^{14,16,17}$ Specifically, the relative timing jitter has to be only a small fraction of the temporal resolution required for the applications. For example, for picosecond CRS microscopy, tens or even hundreds of femtosecond relative timing jitter has virtually no measurable effect on the imaging quality. In contrast, if the relative timing jitter is comparable to the pulse width (i.e., on the order of picosecond), the imaging quality will suffer from dramatic deterioration. ${ }^{4}$

So far, both intrinsic timing jitter of the modelocked laser ${ }^{18}$ and the repetition rate drift of the mode-locked laser ${ }^{19}$ have been theoretically identified as the origins of the relative timing jitter in the synchronized time-lens source. From these results, it seems that a mode-locked laser with perfect timed output, i.e., a pulse train with evenlyspaced optical pulses, would introduce no relative timing jitter. However, Quinlan et al. recently demonstrated theoretically that the shot noise of the optical pulse train from the mode-locked laser, after photodetection, could introduce timing jitter to the electrical pulse train. ${ }^{20}$ Besides, Krune et al. demonstrated that the thermal noise of the photodetector could also introduce timing jitter to the electrical pulse train. ${ }^{21}$ These timing jitters manifest themselves in the fluctuation of "center of mass", i.e., peak positions of the pulse train. As a result, questions naturally arise concerning the synchronized time-lens source, which necessitates $O / E$ conversion with a photodetector at the very beginning of driving electrical signal generation: From the theoretical perspective, is this photodetection a potential cause of the relative timing jitter? If yes, to what extent does this factor affect the synchronization performance? and what measures can we take to suppress the relative timing jitter for the synchronized time-lens source?

In this paper, first we establish a physical model describing the synchronized time-lens source accounting for the noise introduced by Quinlan et al. ${ }^{20}$ and Krune et al. ${ }^{21}$ Next, we demonstrate numerical simulation results to identify that this photodetection-induced timing jitter is a potential origin of the relative timing jitter in the synchronized time-lens source. Through quantitative results, we further show to what extent this jitter source can affect the synchronization performance. Based on the nature of this jitter source, we finally propose several strategies to reduce the relative timing jitter. We expect that these results will provide guidelines for optimizing synchronization performance in experiments.

\section{Theoretical Model and Simulation Details}

\subsection{Timing jitter induced by photodetection}

Both shot noise- ${ }^{20}$ and thermal noise-induced ${ }^{21}$ timing jitters have been explicitly derived with 
analytical forms. Physically, both timing jitters due to the above origins lead to stochastic deviations of the arrival time of the electrical pulses, specifically the "center of mass" positions. In this paper, we introduce these jitters into our model system for the time-lens source. According to Ref. 20, the timing jitters induced by the shot noise of the optical pulse train, before $\left(\sigma_{o}\right)$ and after $\left(\sigma_{e}\right)$ photodetection, are given by the following:

$$
\begin{aligned}
\sigma_{o} & =\sqrt{\frac{1}{8 \ln 2} \frac{h \nu_{c} \tau^{2}}{E_{\mathrm{po}}}}, \\
\sigma_{e} & =\sqrt{\frac{1}{8 \ln 2} \frac{h \nu_{c} \tau^{2}}{\eta E_{\mathrm{pe}}}},
\end{aligned}
$$

where $h$ is Planck's constant, $\nu_{c}$ is the carrier frequency of the incoming light, $\eta$ is the quantum efficiency of the photodetector, $E_{\mathrm{po}}$ is the pulse energy in the mode-locked laser pulse train, $E_{\mathrm{pe}}$ is the pulse energy on the photodetector, and $\tau$ is the full width at half maximum (FWHM) pulse width of the optical pulse assuming a Gaussian intensity profile. We further note that $\sigma_{o}$ is the timing jitter of the mode-locked laser induced by shot noise, and $\sigma_{e}$ is the timing jitter of the electrical pulse train after photodetection. According to both Eqs. (1) and (2), the shot noise will introduce timing jitter to the electrical pulse train after photodetection and $O / E$ conversion.

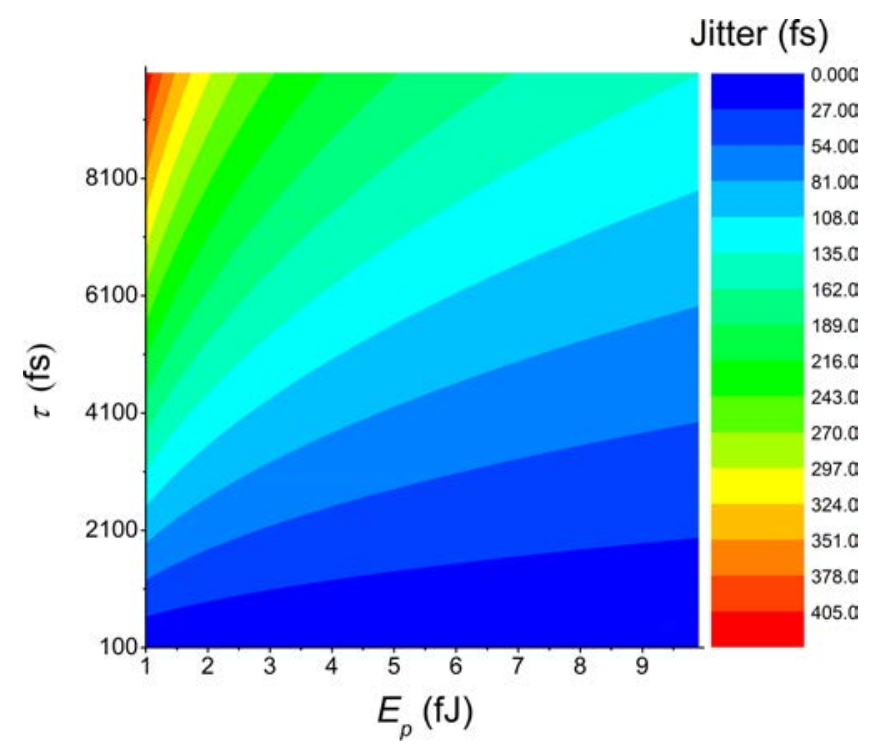

(a)
According to Ref. 21, the timing jitter induced by the thermal noise of the optical pulse train, after photodetection, is given by the following:

$$
\sigma_{\mathrm{th}}=\sqrt{\frac{9.6 k_{B} T \tau_{e}^{3}}{R_{P}^{2} \eta^{2} E_{p}^{2} R_{L}}},
$$

where $k_{B}$ is the Boltzman's constant, $T$ is the temperature of the photodetector, $R_{p}$ is the response of an ideal photodiode, $R_{L}$ is the load resistance, and $\tau_{e}$ is the electrical pulse width after photodetection. According to Eq. (3), the thermal noise will also introduce timing jitter to the electrical pulse train after photodetection and $O / E$ conversion.

Figure 1 exemplifies the timing jitter of the electrical pulse train after photodetection due to shot noise (Fig. 1(a)) and thermal noise (Fig. 1(b)). In the calculation, the following parameters are used: $\nu_{c}$ corresponds to a mode-locked laser center wavelength of $880 \mathrm{~nm}$, and $\eta=2.2 \%$ for the GaAs photodetector at this wavelength. $T=300 \mathrm{~K}$ corresponds to room temperature and $R_{L}=50 \Omega$. From Eqs. (2) and (3) and Fig. 1, it can be clearly seen that the smaller the incoming pulse energy is and the larger the pulse widths are, the higher the timing jitter after photodetection is. If we compare shot noise- and thermal noise-induced jitter quantitatively, we can see that the latter is dominant. We want to note here that, the timing jitter of the mode-locked laser purely due to shot noise

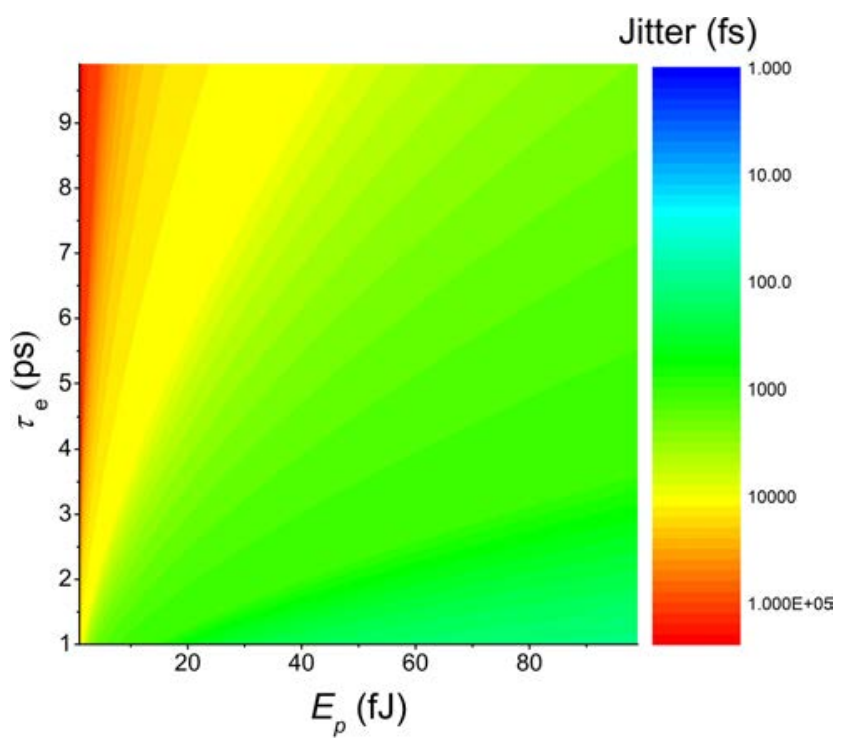

(b)

Fig. 1. Color fill contour plot of the timing jitter of the electrical pulse train due to (a) shot noise and (b) thermal noise as a function of input pulse energy and pulse width. $\tau$ is the optical pulse width and $\tau_{e}$ is the electrical pulse width. 
(Eq. (1)) is quite small. This is because: (1) For the synchronized time-lens source, only a tiny amount of optical power (could be $1 / 1000$ or even smaller) is split off the mode-locked laser output for photodetection. (2) There is no quantum efficiency related degradation of the timing jitter as indicated by Eq. (2). The quantum efficiency of most photodetectors is in the range of $1-50 \%$. Consequently, the optical pulse train from the mode-locked laser is considered to be jitter free, since we confine our discussion here to photodetection-induced timing jitter only.

Our model system of the synchronized time-lens source is similar to that in Ref. 18 (Fig. 1 in Ref. 18). Briefly, a fast photodetector converts the modelocked laser output $(80 \mathrm{MHz})$ into an $\mathrm{RF}$ pulse train of the same repetition rate, which is then divided into two branches. One branch is filtered by a narrowband $(50 \mathrm{MHz}$ unless defined otherwise) $\mathrm{RF}$ filter to get a $10-\mathrm{GHz}$ sine wave to drive the phase modulator, while the other branch $(80 \mathrm{MHz})$ drives the Mach-Zehnder intensity modulator (MZ) to carve a synchronized $80-\mathrm{MHz}$ optical pulse train from the CW laser. After dispersion compensation, the pulse width is compressed to $\sim 1.8 \mathrm{ps}$ for optimal compression. ${ }^{15}$ The key difference is that the incoming mode-locked pulse train is free from any intrinsic timing jitter, and the timing jitter is introduced purely by photodetection. To account for this timing jitter, a Gaussian white noise generator was used to introduce timing jitter of different values calculated by Eq. (2) to the electrical pulse train after photodetection. To calculate the relative timing jitter $\left(\sigma_{\mathrm{syn}}\right)$ between the time-lens source and the mode-locked laser, we first found the peaks and the corresponding temporal positions of the two pulse trains, then do subtraction to get relative jitter, and finally calculate the RMS timing jitter between the two pulse trains. Pulse trains of 64 optical and electrical pulses were simulated.

\section{Simulation Results and Discussion \\ 3.1. Relative timing jitter introduced by photodetection}

First, we elucidate the effect that the timing jitter introduced by photodetection can cause the relative timing jitter between the time-lens source and the mode-locked laser. Throughout the paper, the pulse peak positions are defined as the deviation from their nominal positions without timing jitter. Figures 2(a)-2(c) show the pulse peak positions of the mode-locked laser and the time-lens source, for three typical simulation runs of $300-\mathrm{fs}, 400-\mathrm{fs}$ and 500 -fs electrical timing jitter introduced by photodetection. Since in our simulation, we assumed that the mode-locked laser output is free from any jitter, the peak positions are zero for each pulse in the pulse train. Due to the timing jitter introduced by photodetection, however, the peak positions of the time-lens source show notable deviation from the mod-locked laser. As a result, the relative timing jitter, defined as the peak position difference between the two pulse trains, arises. So, here we identify another origin of relative timing jitter in the synchronized time-lens source system-electrical timing jitter introduced by photodetection.

It can be seen from Fig. 2 that, qualitatively, the higher the timing jitter introduced by photodetection is, the higher the relative timing jitter between the time-lens source and the mode-locked laser is. To get quantitative results, we calculated the dependence of the relative timing jitter on the timing jitter introduced by photodetection, shown in Fig. 3 (red circles). Each data point is the average of ten simulation runs. Overall, the relative timing jitter caused by photodetection is smaller than the timing jitter of the electrical pulse train after photodetection. For example, for $400-f s$ timing jitter on the electrical pulse train, the resultant relative timing jitter is $322 \mathrm{fs}$. As the electrical timing jitter changes from $200 \mathrm{fs}$ to $500 \mathrm{fs}$, the relative timing jitter between the optical pulse trains increases from 159 fs to 405 fs.

\subsection{Comparison to that introduced by the intrinsic timing jitter of the mode-locked laser}

Having identified that photodetection itself is one potential origin of the relative timing jitter, the next question is: Compared with the other identified source of the relative timing jitter, i.e., the intrinsic timing jitter of the mode-locked laser, which one is more severe and causes more relative timing jitter? For quantitative comparison, we also performed numerical simulations assuming an ideal photodetector (which introduces no timing jitter to the detected electrical pulse train) and a mode-locked laser output with intrinsic timing jitter, using the 


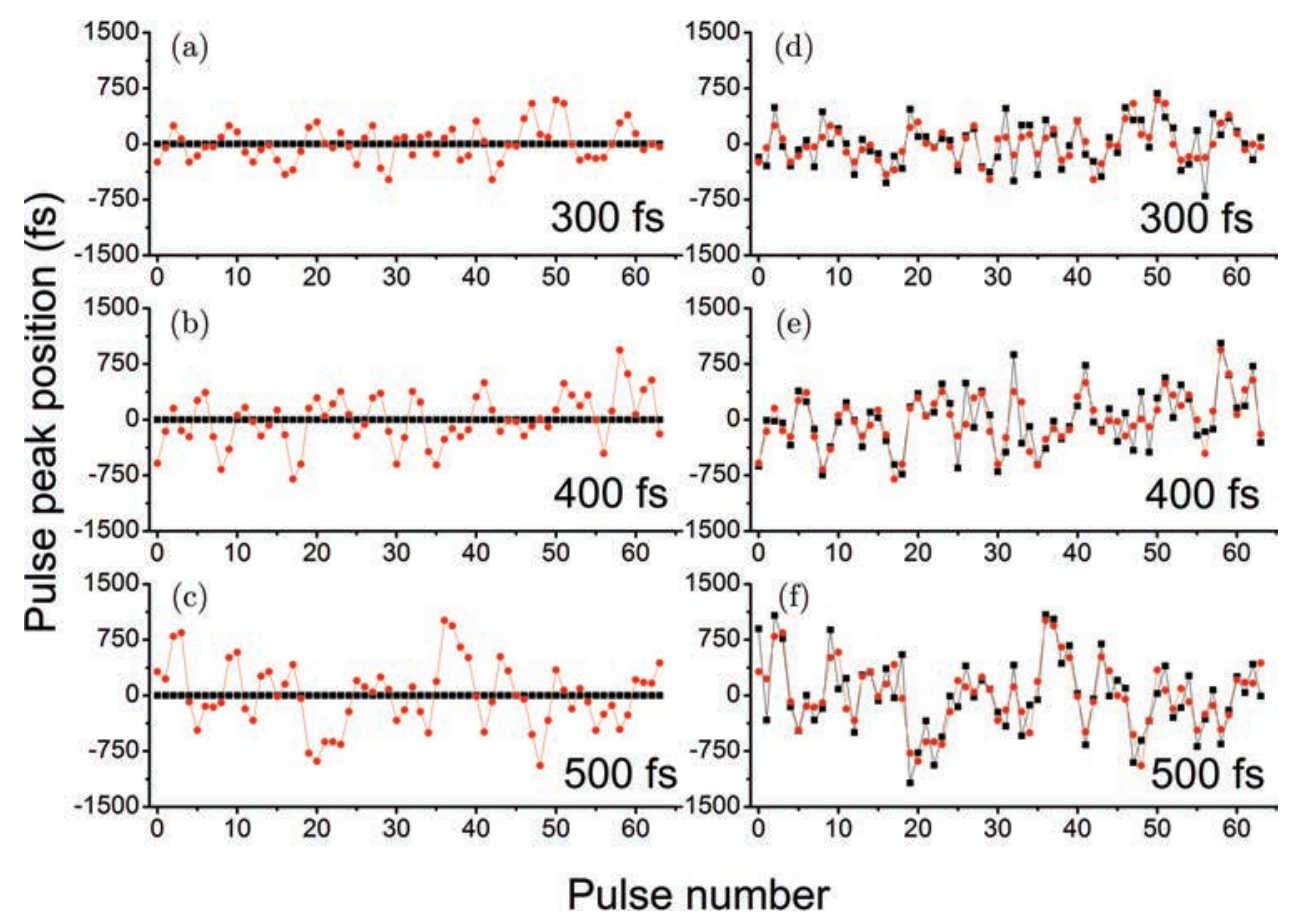

Fig. 2. Pulse peak positions (deviation from their nominal positions without timing jitter) of the mode-locked laser (black squares) and the synchronized time-lens source (red circles) of three typical simulation runs for (a) 300-fs, (b) 400-fs and (c) 500-fs photodetection introduced electrical timing jitter. For comparison, pulse peak positions are also shown for (d) 300-fs, (e) 400-fs and (f) 500-fs intrinsic optical timing jitter in the mode-locked laser.

physical model in Ref. 18 except that a $50-\mathrm{MHz}$ square RF filter was assumed here. Pulse peak positions of three typical runs are shown in Figs. 2(d)2(f). Due to the presence of the intrinsic timing jitter, the pulse peak positions of the mode-locked laser are no longer zero, but show some stochastic

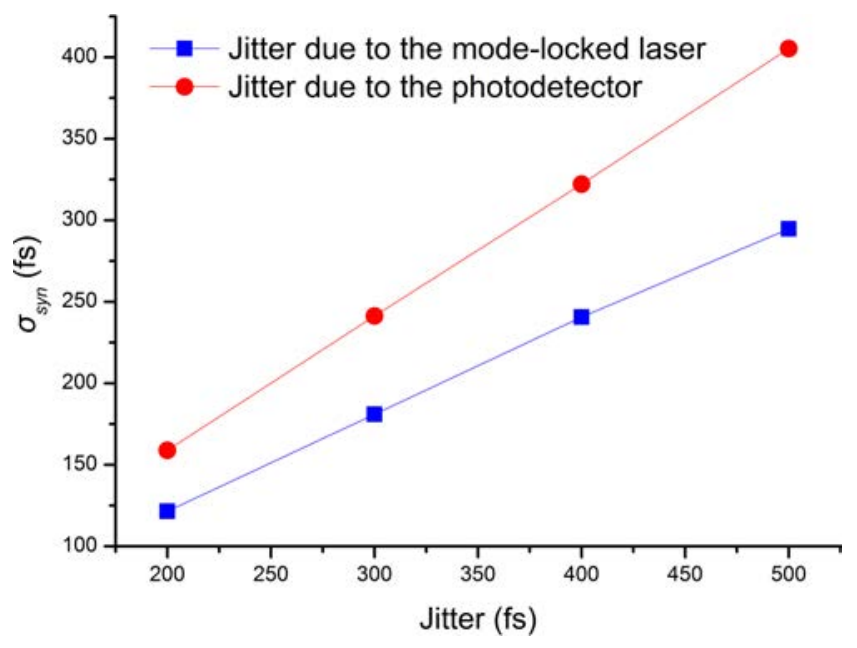

Fig. 3. Relative timing jitter as a function of the timing jitter introduced either by photodetection (red circles), or by the mode-locked laser (blue squares). Each data point is the average of ten successive runs. deviation. However, as shown in Fig. 2 and we mentioned in Ref. 18, the time-lens output can to some extent follows this deviation. As a result, if we calculate the relative timing jitter between the two optical pulse trains, it is even smaller than the case where the relative timing jitter is purely introduced by the photodetector (compared with Figs. 2(a)$2(\mathrm{c})$ ). This can be further confirmed by calculating the dependence of the relative timing jitter on the intrinsic timing jitter of the mode-locked laser, shown by blue squares in Fig. 3. Clearly, for the same amount of timing jitter, the relative timing jitter due to photodetection is more severe than that due to the intrinsic timing jitter of the mode-locked laser. For example, for 400-fs intrinsic timing jitter of the mode-locked laser, the relative timing jitter is $241 \mathrm{fs}, 25 \%$ smaller than that due to the photodetection (322 fs).

\subsection{Strategies to suppress the relative timing jitter due to photodetection}

When the mode-locked laser is free from intrinsic timing jitter, the output pulse train is perfectly timed with the same temporal separation between 


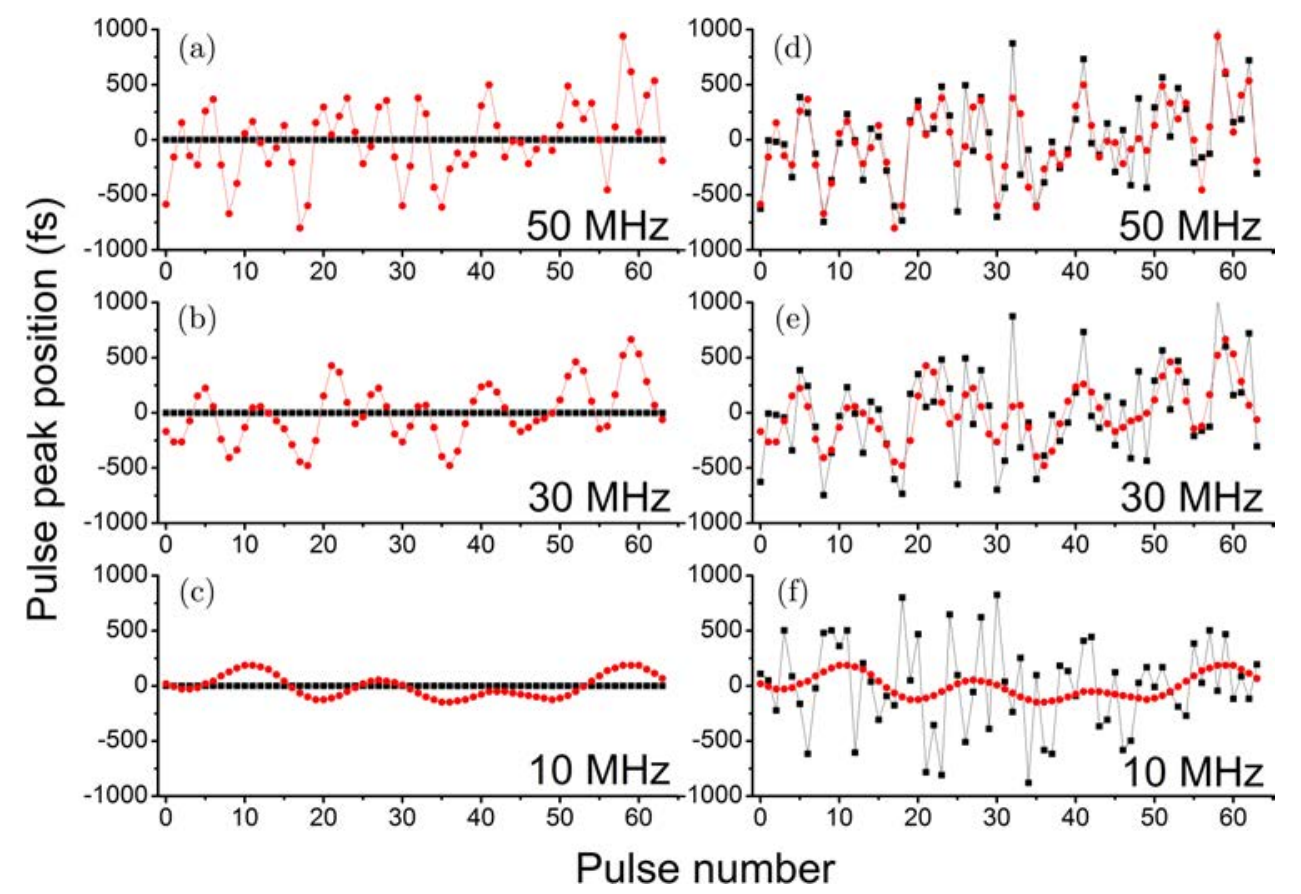

Fig. 4. Pulse peak positions of the mode-locked laser (black squares) and the synchronized time-lens source (red circles) with (a, d) $50-\mathrm{MHz}$, (b, e) 30-MHz and (c, f) 10-MHz RF filter. In (a), (b) and (c), the relative timing jitter is due to the 400-fs electrical timing jitter introduced by the photodetector. In (d), (e) and (f), the relative timing jitter is due to the 400-fs intrinsic timing jitter introduced by the mode-locked laser.

adjacent pulses (Fig. 2). In this case, to suppress the relative timing jitter, the most straightforward strategy is making the time-lens output as close to a perfectly timed pulse train as possible. This can be effectively achieved by narrowing the bandwidth of the RF filter. Figure 4 shows pulse peak positions of the mode-locked laser and the time-lens source. Figures $4(\mathrm{a})-4(\mathrm{c})$ demonstrate that when photodetector-induced jitter is considered, with progressively reduced $\mathrm{RF}$ filter bandwidth from $50 \mathrm{MHz}$ to $30 \mathrm{MHz}$ and then to $10 \mathrm{MHz}$ (assuming all RF filters are square filters and free from group delay dispersion within the passband), the time-lens output gets closer to a perfectly timed pulse train with less deviation from the mode-locked laser. This trend can be quantified by calculating the dependence of relative timing jitter on the filter bandwidth, shown by red circles in Fig. 5. For 400-fs timing jitter introduced by the photodetector, the relative timing jitter is reduced to $135 \mathrm{fs}$ using a $10-\mathrm{MHz} \mathrm{RF}$ filter, $58 \%$ smaller than that using a $50-\mathrm{MHz}$ RF filter (322 fs).

This relative timing jitter suppression technique using narrower filters, however, comes at the cost of increasing the relative timing jitter caused by the

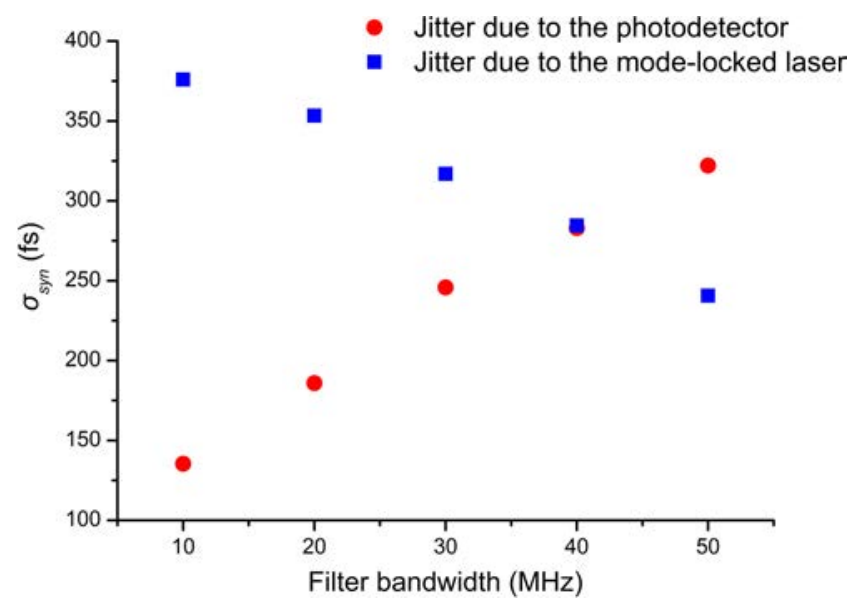

Fig. 5. Relative timing jitter as a function of the RF filter bandwidth. The relative timing jitter is caused either by the 400-fs photodetector induced electrical jitter (red circles), or by the 400-fs mode-locked laser intrinsic timing jitter (blue squares). Each data point is the average of ten successive runs.

intrinsic timing jitter of the mode-locked laser. Figures $4(\mathrm{~d})-4(\mathrm{f})$ show pulse peak positions in the presence of intrinsic timing jitter in the mode-locked laser, instead of photodetector-induced jitter. With progressively decreased filter bandwidth, although 
the time-lens source output pulse train becomes closer to perfectly timed, it deviates more from the jittering mode-locked laser output pulse train, leading to more relative timing jitter between the two pulse trains. This is contrary to the case where the relative timing jitter is introduced by the photodetector. Figure 5 clearly reveals these opposite dependencies on the filter bandwidth. When the filter bandwidth is $40 \mathrm{MHz}$, relative timing jitters due to these two sources are approximately the same ( $\sim 284 \mathrm{fs})$.

The other strategy to reduce the relative timing jitter is to suppress the electrical timing jitter introduced by the photodetector, since the smaller the photodetector jitter is, the smaller the relative jitter is (Fig. 3). In the ideal situation where the photodetector introduces no electrical jitter, the time-lens output will be a perfectly timed pulse train (when the intrinsic timing jitter of the mode-locked laser is not considered), the same as that of the modelocked laser and there will be no relative timing jitter. From the analytical formulas of the photodetector-induced timing jitter, it can be clearly seen that the adjustable experimental parameters are the quantum efficiency $\eta$, the incoming pulse energy $E_{p}$, and the bandwidth of the photodetector $f_{3 \mathrm{~dB}}$. Specifically, $\eta$ can be optimized by resorting to photodetectors with higher quantum efficiency. For example, at $880 \mathrm{~nm}$, the quantum efficiency of InGaAs is $\sim 40 \%, \sim 20$ times higher than that of GaAs (2.2\%). This means switching from GaAs to InGaAs photodetector will reduce the jitter. Increasing the incoming pulse energy $E_{p}$ is an effective strategy to suppress the photodetector jitter. For example, increasing $E_{p}$ from $1 \mathrm{fJ}$ to $1 \mathrm{pJ}$ will reduce the shot noise-induced jitter by 32 times, and the thermal noise-induced jitter by 1000 times, more dramatic than the former due to the $1 / E_{p}$ dependence of the jitter.

\section{Summary and Discussion}

The relative timing jitter between the synchronized time-lens source and the mode-locked laser has to be minimized to extend the applications of this novel synchronized laser system to even shorter timescales below picoseconds. To optimize the synchronization performance, the first problem to be solved is to identify the origins of relative timing jitter. In this paper, through both analytical and numerical investigations, we identify a new origin of the relative timing jitter - the electrical jitter introduced by photodetection. Quantitative results clearly reveal that the higher the photodetector noise is, the higher the resultant relative timing jitter will be, even when the incoming optical pulse train from the mode-locked laser is perfectly timed. Based on the nature of this jitter, we further propose experimental strategies to reduce the relative timing jitter due to the photodetector. Using an RF filter with a narrower bandwidth will reduce the relative timing jitter. Although in the meantime, this will cause an increase in the relative timing jitter due to the intrinsic timing jitter of the mode-locked laser and it is effective irrespective of the magnitude of the detector induce jitter. On the photodetector side, minimizing the electrical jitter induced by the photodetector is also effective in minimizing the relative timing jitter. Among the strategies to this end, i.e., switching to photodetectors with either higher quantum efficiency, and increasing the input pulse energy on the photodetector, the latter is the easiest to implement. We expect these results will provide guidelines for optimizing the synchronization performance of the synchronized time-lens source, facilitating its extension to applications requiring femtosecond synchronized pulses. We further note that there may be other sources of timing jitter, such as thermal noise in electrical amplifiers, amplified spontaneous emission noise in optical amplifiers, group delay dispersion in the chirped fiber Bragg grating (together with fluctuation of the CW laser center wavelength will lead to a fluctuation of the arrival time), and group delay dispersion of the RF filter. These will be the subjects of future investigation.

\section{Acknowledgments}

This work was supported by the National Natural Science Foundation of China (11404218), the Science and Technology Innovation Commission of Shenzhen (JCYJ20160307150657874, KQJSCX20160226194151, KQTD20150710165601017), the Project of Department of Education of Guangdong Province (2014KTSCX114), and the Natural Science Foundation of SZU (00002701). We acknowledge Prof. Ke Wang from Key Laboratory of Optoelectronic Devices and Systems of Ministry of Education and Guangdong Province, College of 
Optoelectronic Engineering, Shenzhen University for helpful discussions.

\section{References}

1. C. Manzoni, R. Osellame, M. Marangoni, M. Schultze, U. Morgner, G. Cerullo, "High-repetitionrate two-color pump-probe system directly pumped by a femtosecond ytterbium oscillator," Opt. Lett. 34, 620-622 (2009).

2. C. W. Freudiger, W. Min, B. G. Saar, S. Lu, G. R. Holtom, C. He, J. C. Tsai, J. X. Kang, X. S. Xie, "Label-free biomedical imaging with high sensitivity by stimulated Raman scattering microscopy," Science 322, 1857-1861 (2008).

3. A. Zumbusch, G. R. Holtom, X. S. Xie, "Three-dimensional vibrational imaging by coherent antiStokes Raman scattering," Phys. Rev. Lett. 82, 4142-4145 (1999).

4. E. O. Potma, D. J. Jones, J.-X. Cheng, X. S. Xie, J. Ye, "High-sensitivity coherent anti-Stokes Raman scattering microscopy with two tightly synchronized picosecond lasers," Opt. Lett. 27, 1168-1170 (2002).

5. D. Zhang, P. Wang, M. N. Slipchenko, D. BenAmotz, A. M. Weiner, J.-X. Cheng, "Quantitative vibrational imaging by hyperspectral stimulated Raman scattering microscopy and multivariate curve resolution analysis," Anal. Chem. 85, 98-106 (2012).

6. K. Wang, C. W. Freudiger, J. H. Lee, B. G. Saar, X. S. Xie, C. Xu, "Synchronized time-lens source for coherent Raman scattering microscopy," Opt. Express 18, 24019-24024 (2010).

7. K. Wang, D. Zhang, K. Charan, M. N. Slipchenko, P. Wang, C. Xu, J. X. Cheng, "Time-lens based hyperspectral stimulated Raman scattering imaging and quantitative spectral analysis," J. Biophoton. 6, 815-820 (2013).

8. K. Wang, C. Xu, "Fiber-delivered picosecond source for coherent Raman scattering imaging," Opt. Lett. 36, 4233-4235 (2011).

9. K. Wang, C. Xu, "Fiber delivered two-color picosecond source through nonlinear spectral transformation for coherent Raman scattering imaging," Appl. Phys. Lett. 100, 071106 (2012).

10. B. Li, K. Charan, K. Wang, T. Rojo, D. Sinefeld, C. $\mathrm{Xu}$, "Nonresonant background suppression for coherent anti-Stokes Raman scattering microscopy using a multiwavelength time-lens source," Opt. Express 24, 26687-26695 (2016).

11. B. H. Kolner, "Active pulse compression using an integrated electrooptic phase modulator," Appl. Phys. Lett. 52, 1122-1124 (1988).

12. K. Wang, Y. Wang, R. Liang, J. Wang, P. Qiu, "Contributed review: A new synchronized source solution for coherent Raman scattering microscopy," Rev. Sci. Instrum. 87, 071501 (2016).

13. J. V. Howe, C. Xu, "Ultrafast optical signal processing based upon space-time dualities," J. Lightwave Technol. 24, 2649-2662 (2006).

14. K. Wang, C. Xu, "Wavelength-tunable high-energy soliton pulse generation from a large-mode-area fiber pumped by a time-lens source," Opt. Lett. 36, 942944 (2011).

15. P. Qiu, K. Wang, "Optimal compression in synchronised time-lens source for CRS imaging," Electron. Lett. 50, 148-149 (2014).

16. Y. Dai, C. Xu, "Generation of high repetition rate femtosecond pulses from a CW laser by a time-lens loop," Opt. Express 17, 6584-6590 (2009).

17. I. Morohashi, T. Sakamoto, H. Sotobayashi, T. Kawanishi, I. Hosako, "Broadband wavelengthtunable ultrashort pulse source using a MachZehnder modulator and dispersion-flattened dispersion-decreasing fiber," Opt. Lett. 34, 2297-2299 (2009).

18. P. Qiu, K. Wang, "Timing jitter in synchronized time-lens source for coherent Raman scattering imaging," Opt. Express 23, 18786-18791 (2015).

19. K. Wang, J. Wang, P. Qiu, "Synchronization maintenance of synchronized time-lens source in the presence of repetition rate drift of the mode-locked laser for coherent Raman scattering microscopy," IEEE J. Quantum Electron. 53, 1-5 (2017).

20. F. Quinlan, T. M. Fortier, H. Jiang, A. Hati, C. Nelson, Y. Fu, J. C. Campbell and S. A. Diddams, "Exploiting shot noise correlations in the photodetection of ultrashort optical pulse trains," Nat. Photon. 7, 290-293 (2013).

21. E. Krune, B. Krueger, L. Zimmermann, K. Voigt, K. Petermann, "Comparison of the jitter performance of different photonic sampling techniques," J. Lightwave Technol. 34, 1360-1367 (2016). 